\title{
Le religioni in città: un contest per le scuole superiori di Bologna
}

L'articolo offre una riflessione sui progetti presentati dalle classi del Liceo Galvani di Bologna in risposta al contest "Le religioni in città" attivato dalla Fondazione per le scienze religiose e dall'European Academy of Religion e rivolto alle scuole superiori. Il contest si inserisce in una serie di progetti promossi da Fscire per il contrasto dell'analfabetismo religioso e intendeva sollecitare gli studenti a una riflessione sul tema del pluralismo e della diversità religiosa da diverse prospettive.

This article offers a review of the projects submitted by the Liceo Galvani's students in response to the contest "Le religioni in città", launched by the Fondazione per le scienze religiose and the European Academy of Religion and addressed to Bologna high schools students. This contest is part of a series of initiatives promoted by Fscire in order to prevent religious illiteracy and it aimed to encourage students to explore from different perspectives the issue of pluralism and religious diversity.

\section{Studi, ricerche e iniziative per il contrasto dell'analfabetismo religioso}

Il contest per le scuole bolognesi Le religioni in città: simboli, luoghi, cibi e persone è stato attivato dalla Fondazione per le scienze religiose (Fscire) e dall'European Academy of Religion in occasione del consueto convegno internazionale dell'associazione europea EuARe, tenutosi a Bologna il 4-7 marzo 2019 e ospitato dalla Fscire. L'obiettivo era quello di coinvolgere direttamente la cittadinanza stimolando una riflessione sulle medesime tematiche che venivano affrontate a 
livello specialistico dagli oltre 1300 partecipanti, che all'interno del convegno hanno analizzato il fenomeno religioso seguendo molteplici punti di vista: storico, sociologico, filosofico, letterario, teologico. Allo stesso tempo, è stata un'occasione per far conoscere un contesto internazionale e accademico ad alcuni cittadini bolognesi, attivando un dialogo e uno scambio fra cittadinanza ed evento scientifico.

Tale iniziativa, strettamente collegata all'occasione del convegno, che ha popolato la città di Bologna delle più autorevoli voci in materia, si inserisce nondimeno in una più ampia riflessione scientifica che Fscire ha avviato sul tema dell'analfabetismo religioso e sulle sue conseguenze per il tessuto sociale e culturale italiano [Melloni 2014] ed europeo [Melloni e Cadeddu 2018]. Attraverso diverse pubblicazioni il tema è stato affrontato a livello scientifico tramite un approccio interdisciplinare, che a partire dalle più recenti indagini statistiche ha proposto riflessioni storiche, sociologiche e giuridiche sulle problematiche causate dal crescente analfabetismo religioso. In particolare, il fenomeno dell'analfabetismo religioso acquisisce una rilevanza sociale in quanto si inserisce in una situazione di sempre più ampio pluralismo religioso, che al contrario richiederebbe alla società e a tutti i suoi attori conoscenze corrette e solide sulla materia. A partire da queste riflessioni Fscire ha dunque attivato diverse attività formative quale un master di primo livello in Pluralismo Religioso e Sapere storico (PluReS), che - grazie anche al sostegno della regione Emilia-Romagna - si propone di fornire a partecipanti provenienti da percorsi formativi e professionali diversi un'introduzione ai testi, alla storia, alle dottrine e alle tradizioni di ebraismo, cristianesimo e islam; oltreché scuole intensive tematiche per la formazione di docenti di scuola. In questo modo ai prodotti della ricerca si sono associati materiali rivolti direttamente a formatori e documenti utili alla divulgazione, la cui diffusione è stata veicolata dalla creazione di un portale online dedicato. Pars, il portale di formazione e informazione per il contrasto dell'analfabetismo religioso, nato grazie a una collaborazione con l'Università di Modena e Reggio Emilia, si propone di diffondere contenuti utili allo studio, alla didattica e all'informazione sul tema delle religioni attraverso la creazione di percorsi di apprendimento e la diffusione di materiali scelti da specialisti.

Il contest per le scuole si è dunque sviluppato all'interno di tale contesto scientifico, formativo e divulgativo al fine di coinvolgere non solo gli attori della formazione, ma andando a interrogarne direttamente i destinatari, cioè gli studenti degli istituti medi superiori della città di Bologna. 
2. Le religioni in città: un contest per dialogare con gli studenti bolognesi

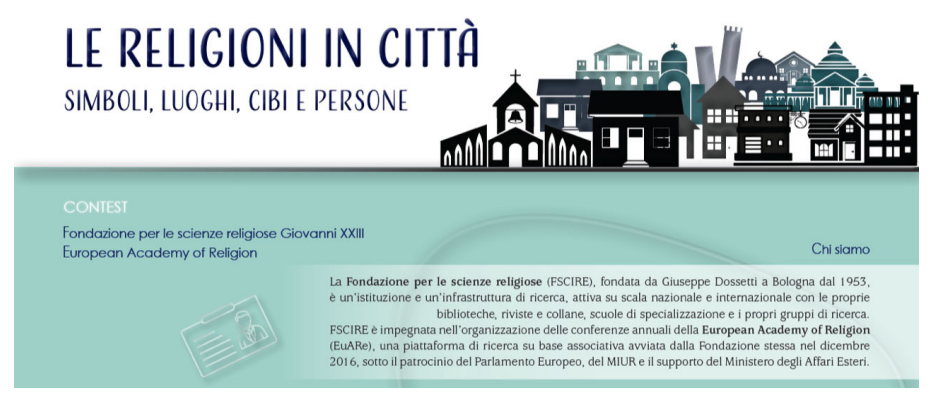

Fig. 1. Locandina contest

Il contest sollecitava dunque classi o singoli gruppi di studenti a ideare gif, cortometraggi, illustrazioni, animazioni, fumetti, fotografie, volti a rappresentare le religioni nella città e nella provincia di Bologna. L'obiettivo del contest era dunque fornire ai docenti di scuola superiore un'occasione e uno stimolo per affrontare il tema del pluralismo religioso e riflettere sulla società bolognese, lasciando libertà al docente di strutturare l'esperienza didattica a seconda della classe e del percorso didattico in corso.

Il premio consisteva in un buono per l'acquisto di libri, consegnato al/alla docente responsabile del progetto, e in un'intervista ai vincitori e alle vincitrici del contest, trasmessa all'interno della rubrica televisiva Il Sabbatico, in onda tutti i sabati su Rainews. In tal modo si intendeva sia promuovere l'approfondimento di tali tematiche, sostenuto anche dall'acquisto di libri, sia dare visibilità presso un pubblico più ampio al pluralismo che caratterizza il tessuto sociale bolognese e la sua istituzione scolastica.

La vittoria è stata assegnata al gruppo della $2^{\mathrm{a}} \mathrm{Q}$ del Liceo ginnasio Luigi Galvani di Bologna che ha presentato il cortometraggio Religioni al femminile; ma tutti i gruppi classe del Liceo Galvani, coordinati dal prof. Francesco Paolo Monaco, sono stati invitati a partecipare alla rubrica televisiva per poter presentare le diverse suggestioni emerse dagli approfondimenti proposti dagli studenti a partire da una riflessione sul fenomeno religioso in città. Il modo migliore per presentare i risultati e il lavoro fatto è sicuramente lasciar parlare direttamente i progetti stessi. 


\section{I risultati: i progetti del liceo Galvani di Bologna}

Tutti i progetti che sono stati presentati hanno mostrato come, a partire da un argomento specifico e da una suggestione piuttosto semplice, i gruppi di studenti siano stati in grado di sviluppare tematiche diverse e complesse: il ruolo delle donne, il tema dei diritti per l'infanzia, la geografia e le caratteristiche dei luoghi di culto.

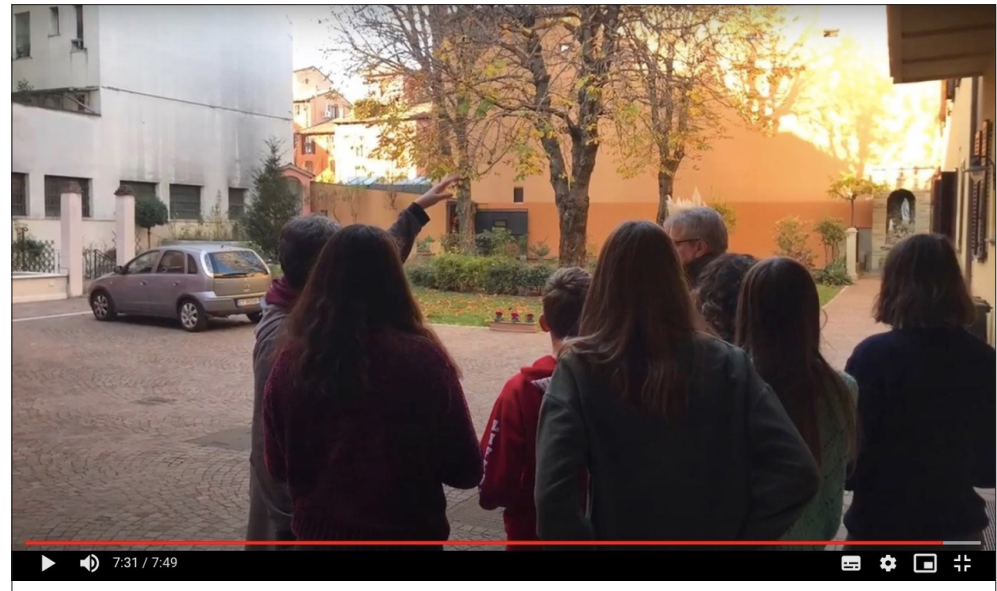

Fig. 2. "Le religioni al femminile", progetto classe $2^{a} Q$ Liceo Galvani

Il gruppo della classe $2^{\mathrm{a}} \mathrm{Q}$ si è aggiudicato la vittoria del contest grazie a un video intervista dal titolo Le religioni al femminile. Il lavoro è stato frutto di una riflessione del gruppo sul ruolo e i diritti della donna nella nostra società. Nonostante le conquiste di parità formale ottenute nei decenni passati, osservano gli studenti:

sul piano sostanziale, però, le statistiche ancora mostrano che in molte zone, anche di Paesi come il nostro, le donne ancora percepiscono stipendi più bassi dei maschi a parità di ruolo, sono più chiamate a far fronte a cure domestiche rispetto ai mariti, sono molto più spesso degli uomini vittime di fenomeni di violenza domestica, che talvolta conducono al femminicidio (v. anche ultimi episodi di cronaca italiana in merito), che sta diventando un'autentica piaga sociale.

Le ragazze e i ragazzi si sono interrogati dunque su se e come è cambiato il ruolo delle donne nelle religioni e se ci sono ancora resistenze a riconoscerne pari dignità negli ambiti in cui si svolge la vita dei credenti. Per cercare risposte a tali quesiti hanno scelto di interrogare le dirette interessate, attraverso la realizzazione di interviste a cinque giovani donne, alle quali è stato chiesto come percepiscono la loro posizione e il ruolo femminile nella propria religione. Le interviste presentano così le diverse visioni e letture della società e della propria comunità 
di fede - ebraica, cattolica, musulmana e ortodossa - nel contesto specifico della città di Bologna.

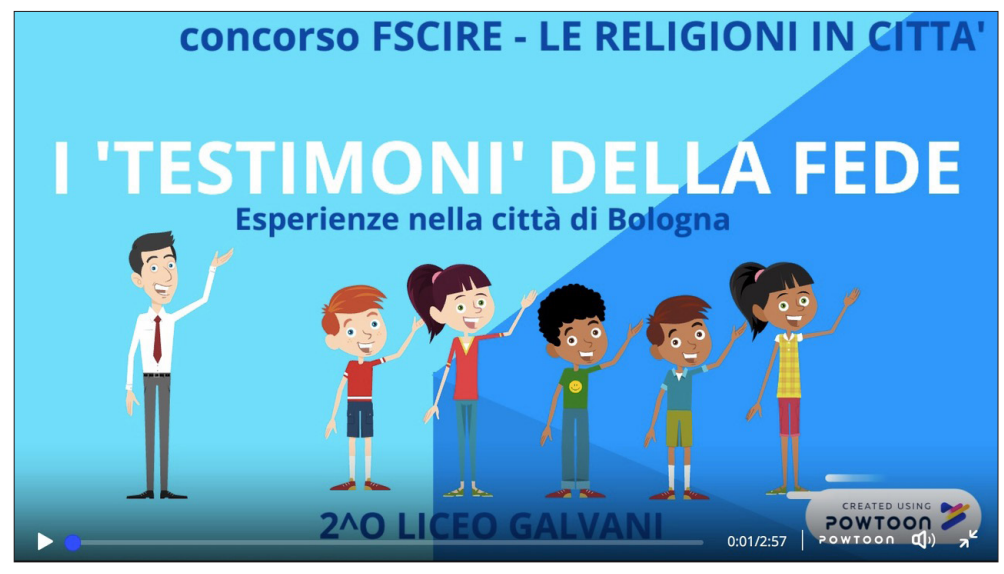

Fig. 3. "I

testimoni della fede", progetto classe $2^{a} \mathrm{O}$ Liceo Galvani

La classe $2^{\mathrm{a}} \mathrm{O}$ ha invece sviluppato le sue riflessioni scegliendo il tema del ruolo dell'infanzia nelle religioni, associandolo alla recente Agenda 2030 pubblicata dall'Onu. Il progetto, presentato con il titolo I testimoni della fede, si apre con un'analisi del programma per lo sviluppo sostenibile dell'Organizzazione delle Nazioni Unite, che include l'obiettivo 16 su pace, giustizia e istituzioni solide. All'interno del documento la classe ha individuato alcuni punti su infanzia ed educazione, il cui fondamento è stato rinvenuto in quattro principi fondamentali espressi nella Convenzione sui diritti dell'infanzia e dell'adolescenza del 1991. Il gruppo si è dunque interrogato sul diritto all'educazione, ragionando sul ruolo delle religioni in questo processo. I ragazzi hanno cercato enti che operassero in tale ambito per valutare le iniziative promosse e hanno scelto di soffermarsi sul documento dell'Ong Arigatou International Faith and Children's Rights. A Multi-religious Study on the Convention on the Rights of the Child, che analizza il ruolo delle religioni per l'educazione e la promozione dei diritti dell'infanzia. Gli studenti, quindi, hanno creato un vero e proprio corpus documentario sulla base del quale avviare una discussione comune; per rispondere ai quesiti emersi hanno scelto di ricercarsi «presso una chiesa cattolica,

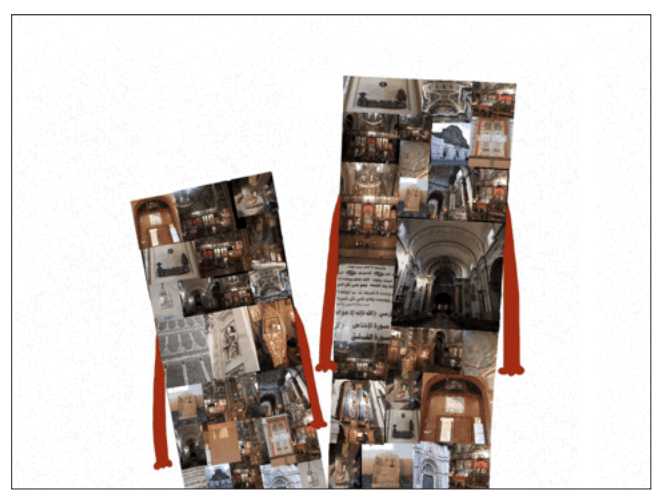

Fig.4. "Le braccia siamo Noi", progetto $3^{a} \mathrm{~F}$ Liceo Galvani 
una chiesa ortodossa moldava e un centro islamico a Bologna, per intervistare $\mathrm{i}$ responsabili delle diverse comunità, come 'testimoni'».

Le interviste ai "testimoni" sono state proposte attraverso una presentazione animata, che ha consentito di integrare le informazioni estratte dai documenti ufficiali analizzati con le voci degli intervistati, che calavano tali punti programmatici nel contesto delle comunità di fede bolognesi.

Se sicuramente l'esperienza spaziale e materiale ha caratterizzato tutti i progetti che per diverse ragioni hanno attraversato la città, è però il lavoro Le braccia siamo Noi che mette al centro del progetto questo aspetto. Il risultato di tale lavoro è stato la creazione di un gif, che mette insieme le fotografie scattate durante le visite fatte ai diversi luoghi di culto bolognesi: la cattedrale di San Pietro e la basilica di San Petronio, la chiesa russa di San Basilio e il centro islamico. Il processo di creazione della Gif, una volta rientrati dalle visite, viene così descritto dagli stessi studenti della $3^{\mathrm{a}} \mathrm{F}$ :

ci siamo confrontati e abbiamo deciso che avremmo utilizzato le due torri, il simbolo della nostra città, e sostituito i mattoni con immagini significative e simboliche di ogni singola professione di fede. Abbiamo pensato che per simboleggiare l'armonia che le caratterizza, le due torri fossero troppo lontane. Come fare per mostrare questa loro caratteristica? La soluzione migliore che abbiamo trovato è stata quella di farle "abbracciare". L'abbraccio per noi simboleggia la fraternità, che si esprime concretamente nel riconoscere l'alterità, l'accoglienza, il dare e ricevere e costruire così un dialogo fruttuoso.

Diversamente dai progetti precedentemente presentati, che si focalizzano sulle diverse comunità religiose $\mathrm{e} i$ rispettivi edifici di culto, il lavoro presentato da alcuni studenti della classe $2^{\mathrm{a}} \mathrm{F}$ enfatizza il ruolo centrale della scuola. Nel corso di un breve fumetto gli studenti raccontano come ragazzi che professano religioni diverse - mostrandone anche la dimensione più intima e domestica della preghiera - possano incontrarsi sui banchi di scuola. Con poche immagini gli studenti esprimono l'importanza per loro dell'istituzione scolastica, che in quanto spazio

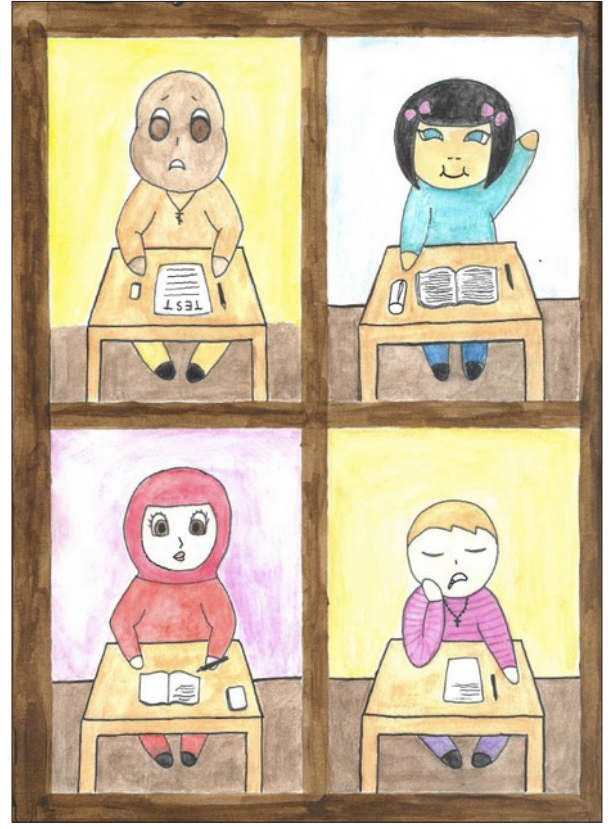

Fig. 5. Progetto classe $2^{a} F$ Liceo Galvani 
pubblico condiviso, consente a ragazzi che provengono da culture e tradizioni differenti di confrontarsi.

Diversi sono dunque i mezzi e i supporti comunicativi scelti - la video-intervista, la presentazione animata, la Gif e il fumetto - alla ricerca di quello più appropriato per veicolare il proprio messaggio e mostrare le religioni in città. Ciò che sicuramente accomuna tutti i progetti qui presentati è però un approfondimento di tematiche complesse grazie alla ricerca di fonti, all'avvio di una riflessione collettiva e a una fase di scoperta del territorio e dei suoi attori prima dell'elaborazione del progetto. Ed è grazie al lavoro svolto in classe che è possibile intravedere nei lavori presentati, quali siano le tematiche che più interessano e interrogano gli studenti rispetto alla società in cui vivono e al fenomeno religioso: il ruolo femminile, la tutela dei diritti dei minori, la conoscenza e il rispetto di culture diverse e il ruolo dell'istituzione scolastica.

\section{Bibliografia}

Melloni A. (ed.) 2014, Rapporto sull'analfabetismo religioso in Italia, Bologna: Il Mulino Melloni A. e Cadeddu F. (eds.) 2019, Religious Literacy, Law and History: Perspectives on European Pluralist Societies, London: Routledge

\section{Risorse}

Portale di formazione e informazione per il contrasto dell'analfabetismo religioso - Pars https://www.pars-edu.it

Le religioni in città: simboli, luoghi, cibi e persone - locandina. pdf https:/e-review.it/sites/default/images/articles/media/233/Le religioni in città simboli, luoghi, cibi e persone - locandina.pdf 
